\title{
Data Science for Finite Strain Mechanical Science of Ductile Materials
}

\author{
Modesar Shakoor \\ Orion L. Kafka \\ Cheng $\mathrm{Yu}$ \\ Wing Kam Liu* \\ Department of Mechanical Engineering, Northwestern University \\ Evanston, IL 60208, USA
}

April 29, 2019

\begin{abstract}
A mechanical science of materials, based on data science, is formulated to predict process-structure-propertyperformance relationships. Sampling techniques are used to build a training database, which is then compressed using unsupervised learning methods, and finally used to generate predictions by means of supervised learning methods or mechanistic equations.

The method presented in this paper relies on an a priori deterministic sampling of the solution space, a K-means clustering method, and a mechanistic Lippmann-Schwinger equation solved using a self-consistent scheme. This method is formulated in a finite strain setting in order to model the large plastic strains that develop during metal forming processes. An efficient implementation of an inclusion fragmentation model is introduced in order to model this micromechanism in a clustered discretization.

With the addition of a fatigue strength prediction method also based on data science, process-structureproperty-performance relationships can be predicted in the case of cold-drawn NiTi tubes.
\end{abstract}

\section{Introduction}

Increasing research efforts in fine scale experiments and numerical modeling in recent decades have progressively led to a change in modeling approaches in mechanics and materials science. Empirical and phenomenological material laws that were previously used to model the nonlinear mechanical response of structures and materials are being replaced by microstructure-based mechanistic material laws.

Under arbitrary loading conditions the number of microstructure observations and conditions to be modeled make the effort required for such an endeavor untenable for practical applications. The appeal of data science and in particular machine learning is a drastic reduction in the number of microstructure observations and simulations required to generate predictive material laws. There is hence a great interest in a data science theory for mechanical science of materials that could generate predictive material laws from a predefined database of experimental and numerical results.

Multiple approaches have been proposed in the literature to reach this goal, generally summarized by these three steps:

1. Collect data using high-fidelity experiments and simulations to build a training database.

2. Compress the training database using unsupervised learning methods for dimension reduction.

*Corresponding author: w-liu@northwestern.edu 
3. Generate predictions using supervised learning methods or mechanistic equations on the compressed training database and optionally cross-validating those predictions using a testing database with new high-fidelity experiments and simulations.

The training database can be generated using, e.g., random sampling [Goury et al., 2016], Gaussian processes [Goury et al., 2016], or Sobol sequences [Bessa et al., 2017]. Because those sampling methods may require a lot of data points to cover the solution space sufficiently for accurate predictions, deterministic sampling methods have been considered by some authors [Yvonnet \& He, 2007, Liu et al., 2016]. For instance, instead of considering a large number of arbitrary, random loading conditions for the training database, only 6 orthogonal loading conditions of small amplitude were proved to be sufficient for small strain elastoplastic analysis in [Liu et al., 2016].

Compression of the training database can be achieved using various unsupervised learning methods for dimension reduction, such as Proper Orthogonal Decomposition (POD) [Ryckelynck, 2005, Lieu et al., 2006, Yvonnet \& He, 2007, Kerfriden et al., 2011, Goury et al., 2016], K-means clustering [Liu et al., 2016, Liu et al., 2018a, Liu et al., 2018b, Kafka et al., 2018] and self-organizing maps [Tang et al., 2018]. The choice of compression method has a significant importance as it defines the discretization of mechanistic equations that will be solved in the prediction stage. POD leads to shape functions of global support, while clustering methods ensure a cluster-wise discretization.

As a result of data compression, the complexity of high-fidelity experiments and simulations that were used to build the training database is encapsulated in a few degrees of freedom. In order to solve for those degrees of freedom and predict mechanical response at arbitrary loading conditions, mechanistic equations have to be reformulated in terms of the reduced degrees of freedom. This new formulation of mechanistic equations is usually called a reduced order model, although this denomination encompasses approaches such as proper generalized decomposition [Ladevèze et al., 2010, Chinesta et al., 2013] which do not rely on data science. Additionally, some approaches couple the data compression and mechanistic prediction steps to improve the reduced order model during the simulation [Ryckelynck, 2005, Kerfriden et al., 2011]. Some supervised learning methods have been applied directly to the training database with a built-in compression stage. This is the case for instance for artificial neural networks, which have been applied in the literature to predict mechanical properties of materials as a function of their microstructural characteristics [Zhang \& Friedrich, 2003, Hambli et al., 2011, Le et al., 2015].

In this paper, we will revisit Self-consistent Clustering Analysis (SCA), a data-driven mechanistic material modeling theory that has been recently developed for small strain elastoplastic materials [Liu et al., 2016]. SCA relies on data compression through clustering and mechanistic prediction through micromechanics and homogenization theory.

Convergence of SCA was proved theoretically recently in [Tang et al., 2018]. Other contributions have recently shown the capability of SCA to account for complex behavior of the microstructure's constituents by embedding crystal plasticity (CP) material laws [Liu et al., 2018b, Kafka et al., 2018] or continuum damage models [Liu et al., 2018a].

The method is herein revisited as a data science mechanistic approach and extended to ductile materials. To reach such an end, the mechanistic equations that SCA relies on to make predictions are reformulated for finite strain elastoplastic materials in Sec. 2. Numerical convergence of this new method is verified in Sec. 3. This new formulation of SCA enables the prediction of the nucleation of voids in ductile materials by debonding and fragmentation of inclusions at the scale of their microstructure, which is shown in Fig. 1. This prediction is achieved with a complexity reduced by several orders. This advantage is exploited in Sec. 4 to predict process-structure-property relations for cold drawn Nickel-Titanium (NiTi) tubes. 


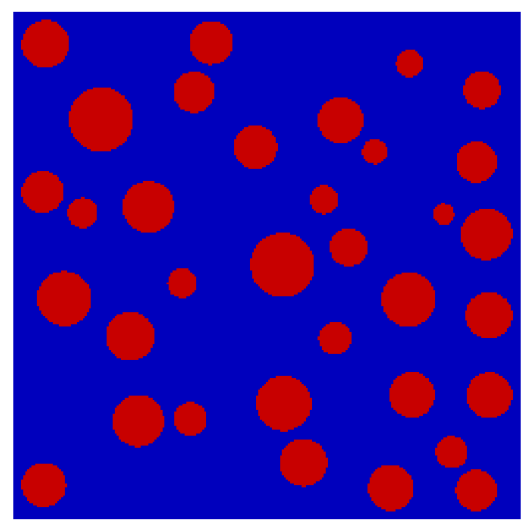

(a)

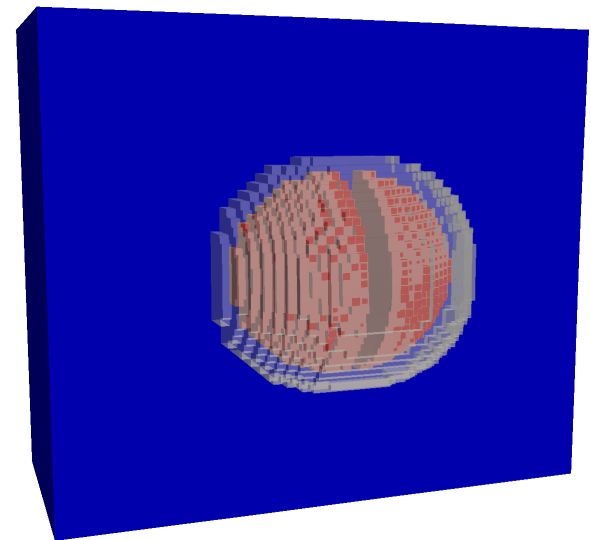

(b)

Figure 1: Ductile materials' microstructures discretized using voxel meshes with matrix shown in blue and inclusions in red: (a) two-dimensional microstructure, (b) inside view of a three-dimensional microstructure with a fragmented inclusion surrounded by a debonding void shown in light gray.

\section{Data science formulation}

Microstructure-based material modeling requires the definition of an idealistic or statistically representative microstructure realization, called Representative Volume Element (RVE). Homogenized material laws can be computed by analytically or numerically solving a boundary value problem for the response of that RVE. For arbitrary microstructure geometries and complex behavior of microstructure constituents (plasticity, fracture), numerical methods such as the Finite Element (FE) method or Fast Fourier Transform (FFT)based numerical methods [Moulinec \& Suquet, 1998] are required.

The microstructures that will be studied in the present paper correspond to ductile materials and feature one or multiple inclusions and voids embedded in a matrix, as shown in Fig. 1. The complexity of the microstructure's constituents' behavior arises due to the hyperelastoplastic response of the matrix, the hyperelastic-brittle behavior of the inclusions, and debonding micromechanisms at the matrix/inclusions interface.

The FE method can be used with any structured or unstructured FE mesh of the undeformed RVE domain $\Omega_{0}^{m}$ (the superscript $m$ means microscopic), while FFT-based methods require structured voxel meshes such as that shown in Fig. 1. In the FE method discrete equations are written for the displacement field $\boldsymbol{u}^{m}$, which is approximated at mesh nodes as

$$
\boldsymbol{u}^{m}(\boldsymbol{X}) \approx \sum_{n=1}^{N_{\text {nodes }}} \boldsymbol{u}^{m, n} N^{n}(\boldsymbol{X}), \boldsymbol{X} \in \Omega_{0}^{m},
$$

where $N_{\text {nodes }}$ is the number of nodes in the FE mesh, $\boldsymbol{u}^{m, n}$ is the displacement vector at node $n$, and $N^{n}$ is the FE shape function at node $n$. In FFT-based numerical methods, discrete equations are written for the deformation gradient tensor field $\mathbf{F}^{m}=\mathbf{I}+\nabla_{\boldsymbol{X}} \boldsymbol{u}^{m}$, which is approximated voxel-wise as

$$
\mathbf{F}^{m}(\boldsymbol{X}) \approx \sum_{n=1}^{N_{\mathrm{voxels}}} \mathbf{F}^{m, n} \chi^{n}(\boldsymbol{X}), \boldsymbol{X} \in \Omega_{0}^{m},
$$

where $N_{\text {voxels }}$ is the number of voxels, $\mathbf{F}^{m, n}$ is the deformation gradient tensor in voxel $n$, and $\chi^{n}(\boldsymbol{X})$ is the characteristic function which is equal to 1 if $\boldsymbol{X}$ is inside voxel $n$ and zero otherwise. 
For a given microstructure, the displacement field $\boldsymbol{u}^{m}$ and the deformation gradient field $\mathbf{F}^{m}$ depend on boundary conditions applied to the RVE. In the present work, $\boldsymbol{u}^{m}$ will be decomposed over the RVE domain $\Omega_{0}^{m}$ into a linear part and a periodic part. As a result, $\mathbf{F}^{m}$ will be decomposed into a constant part $\mathbf{F}^{M}$ (the superscript $M$ means Macroscopic) and a periodic part with zero average over $\Omega_{0}^{m}$. These assumptions correspond to first order homogenization theory [Moulinec \& Suquet, 1998, Geers et al., 2010].

Data science is used in the mechanical science of materials to predict either $\boldsymbol{u}^{m}$ or $\mathbf{F}^{m}$ as a function of $\mathbf{F}^{M}$. As stated in the introduction, the first step is to generate data through simulations. Following [Liu et al., 2016], this will be done using a priori sampling of loading conditions in Secs. 3 and 4.

Simulation results in the training database will have large dimensions due to dependence of approximations in Eqs. (1) and (2) on either the number of nodes or the number of voxels. Data compression is necessary to obtain new approximations with reduced dimensions.

\subsection{Data compression}

Dimension reduction can be achieved using various methods among which POD and clustering are presented and compared in the following.

The general formulation of data science approaches that is developed herein is only relevant if the complexity of simulations that are to be conducted in the prediction stage is at least one order superior to the complexity of simulations required in the training and data compression stage. The relevance of data science approaches also depends on the amount of work that can be transferred out of the prediction stage. This will be evidenced in the following in the case of POD and clustering based data science approaches for mechanical science of materials.

Data compression in the case of POD consists in replacing the large number of local FE shape functions $\left(N^{n}\right)_{n=1 \ldots N_{\text {nodes }}}$ by $K \ll N_{\text {nodes }}$ global functions $\left(\boldsymbol{W}_{i}^{k}\right)_{k=1 \ldots K, i=1 \ldots 3}$, called principal components or modes. The latter can be computed using various decomposition techniques such as principal component analysis or singular value decomposition [Liang et al., 2002]. The resulting approximation replacing Eq. (1) is

$$
u_{i}^{m}(\boldsymbol{X}) \approx \sum_{k=1}^{K} u_{i}^{m, k} \boldsymbol{W}_{i}^{k}(\boldsymbol{X}), \boldsymbol{X} \in \Omega_{0},
$$

where the modes are discretized at mesh nodes as

$$
\boldsymbol{W}_{i}^{k}(\boldsymbol{X})=\sum_{n=1}^{N_{\text {nodes }}} W_{i}^{k, n} N^{n}(\boldsymbol{X}), \boldsymbol{X} \in \Omega_{0} .
$$

Simulations in the prediction stage can then be conducted using a standard FE weak form but replacing approximation (1) by (3). It is interesting to see that once the modes are computed in the data compression stage, Eq. (4) can be precomputed at integration points of the FE mesh in the same way that FE shape functions are usually precomputed in FE codes [Ryckelynck, 2005, Yvonnet \& He, 2007, Kerfriden et al., 2011]. However, if the material is heterogeneous, or if it has a nonlinear behavior that leads to heterogeneous deformations, material integration still has to be solved at each integration point [Ryckelynck \& Missoum Benziane, 2010]. Consequently, in the POD method, the complexity of material integration is not reduced. Additionally, the stiffness matrix associated to the FE weak form is dense because of the form of Eq. (4), and hence its solution using direct or iterative solvers has a cubic worst-case complexity instead of quadratic. However, this complexity depends on $K$ instead of $N_{\text {nodes }}$, with $K \ll N_{\text {nodes}}$, and is therefore drastically reduced by POD.

Data compression in the case of SCA follows a different approach, where the initial numerical method is FFT-based. The large number of voxels is to be replaced by $K \ll N_{\text {voxels }}$ mutually-exclusive groups of voxels that are called clusters and that span the entire RVE domain. Clusters can be constructed using various clustering techniques such as K-means clustering [Liu et al., 2016, Liu et al., 2018a, Liu et al., 2018b, 
Kafka et al., 2018], or self-organizing maps [Tang et al., 2018]. Examples of data that can be used for clustering are given in Sec. 3. The resulting approximation replacing Eq. (2) is

$$
\mathbf{F}^{m}(\boldsymbol{X}) \approx \sum_{k=1}^{K} \mathbf{F}^{m, k} \chi^{k}(\boldsymbol{X}), \boldsymbol{X} \in \Omega_{0}^{m},
$$

where $\mathbf{F}^{m, k}$ is the cluster-wise constant deformation gradient tensor in cluster $k$, and $\chi^{k}(\boldsymbol{X})$ is the characteristic function which is equal to 1 if $\boldsymbol{X}$ is inside any voxel of cluster $k$, and zero otherwise. Because in the FFT-based numerical method the degrees of freedom are directly the voxel-wise constant deformation gradients [Moulinec \& Suquet, 1998], interpolation and integration are carried out at the same points. Thus, clustering degrees of freedom directly leads to a reduction of the number of degrees of freedom and of material integration complexity. In fact, in SCA, the complexity of all operations conducted in the prediction stage only depends on the number of clusters $K$, with the most expensive operation being, similarly to POD, the solution of a dense linear system. The latter results from the reformulation and discretization of the Cauchy equation into the discrete Lippmann-Schwinger equation. These steps are described in the following in the finite strain case following recent work on finite strain FFT-based numerical methods [Kabel et al., 2014] and then integrating it into SCA [Liu et al., 2016].

\subsection{Continuous Lippmann-Schwinger equation}

As mentioned previously, first order homogenization consists in defining the deformation gradient tensor field in the RVE $\mathbf{F}^{m}$ as the addition of the macroscopic (homogeneous) deformation gradient $\mathbf{F}^{M}$ and a microscopic (heterogeneous) fluctuation. Hill's lemma can be used to define the macroscopic first Piola-Kirchhoff stress tensor $\mathbf{P}^{M}$ as the average of the microscopic one $\mathbf{P}^{M}=\frac{1}{\left|\Omega_{0}^{m}\right|} \int_{\Omega_{0}^{m}} \mathbf{P}^{m}(\boldsymbol{X}) \mathrm{d} \boldsymbol{X}$ [Geers et al., 2010].

Hill's lemma requires $\left(\mathbf{F}^{m}-\mathbf{F}^{M}\right)$ to verify compatibility, i.e., to derive from a periodic displacement field, and $\mathbf{F}^{m}$ to verify equilibrium, i.e. to be the solution of the Cauchy equation

$$
\nabla_{\boldsymbol{X}} \cdot \mathbf{P}^{m}\left(\mathbf{F}^{m}(\boldsymbol{X})\right)=0, \boldsymbol{X} \in \Omega_{0}^{m} .
$$

It can be shown [Kabel et al., 2014] that Eq. (6) is equivalent to the Lippmann-Schwinger equation

$$
\mathbf{F}^{m}(\boldsymbol{X})=-\int_{\Omega_{0}^{m}} \mathbb{G}^{0}\left(\boldsymbol{X}, \boldsymbol{X}^{\prime}\right):\left(\mathbf{P}^{m}\left(\mathbf{F}^{m}\left(\boldsymbol{X}^{\prime}\right)\right)-\mathbb{C}^{0}: \mathbf{F}^{m}\left(\boldsymbol{X}^{\prime}\right)\right) \mathrm{d} \boldsymbol{X}^{\prime}+\mathbf{F}^{0}, \boldsymbol{X} \in \Omega_{0}^{m}
$$

The fourth rank tensor $\mathbb{C}^{0}$ is the stiffness tensor associated to an isotropic linear elastic reference material. It will be determined in Subsec. 2.3, as well as the far field deformation gradient tensor $\mathbf{F}^{0}$ and the periodic Green's operator $\mathbb{G}^{0}$. The latter maps any tensor field $\tau^{m}$ to a compatible one:

$$
\exists \boldsymbol{u} \in\left(H^{1}\left(\Omega_{0}^{m}\right)\right)^{3}, \mathbf{u} \text { periodic on } \Omega_{0}^{m},-\mathbb{G}^{0} * \boldsymbol{\tau}^{m}=\nabla_{\boldsymbol{X}} \boldsymbol{u},
$$

where $H^{1}\left(\Omega_{0}^{m}\right)$ is the Sobolev space of square-integrable functions whose weak derivatives are also squareintegrable.

The combination of Eqs. (7) and (8) yields a microscopic deformation gradient tensor $\mathbf{F}^{m}$ that verifies compatibility and a first Piola-Kirchhoff stress tensor $\mathbf{P}^{m}$ that verifies equilibrium.

\subsection{Discrete Lippmann-Schwinger equation}

SCA consists of solving Eq. (7) cluster-wise instead of voxel-wise. This choice is inspired from micromechanics and in particular Transformation Field Analysis [Dvorak, 1992]. Fig. 2 shows an example of clustering performed on the microstructures in Fig. 1. 


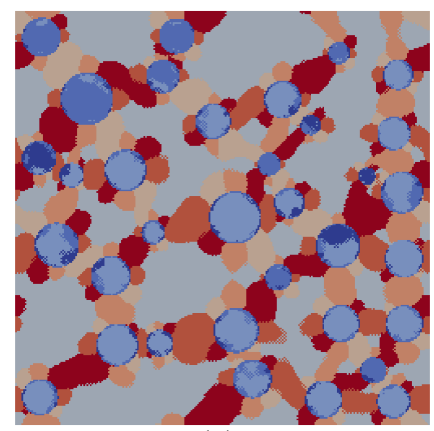

(a)

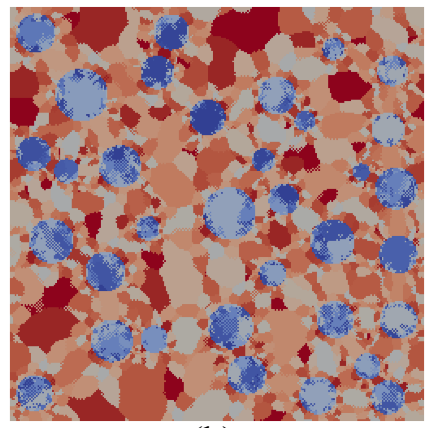

(b)

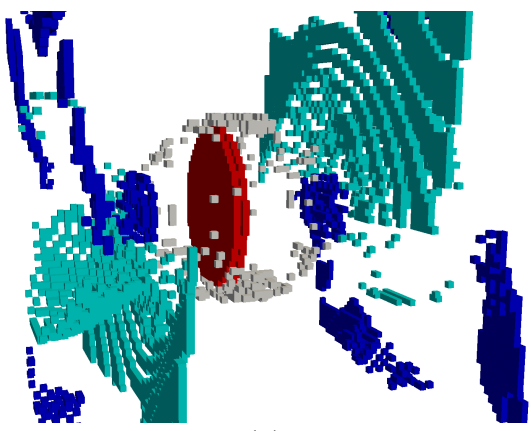

(c)

Figure 2: Ductile materials' microstructures: (a) two-dimensional microstructure discretized using 8 clusters, (b) same two-dimensional microstructure discretized using 65 clusters, (c) three-dimensional microstructure discretized using 217 clusters showing two clusters in the matrix phase (two shades of blue), one cluster in the inclusion phase (red), and one cluster in the void phase (light gray).

As a result of the training stage, the RVE domain $\Omega_{0}^{m}$ is discretized into $K$ subsets $\left(\Omega_{0}^{m, k}\right)_{k=1 \ldots K}$. The degrees of freedom in the FFT-based numerical method [Moulinec \& Suquet, 1998] are associated with the microscopic deformation gradient $\mathbf{F}^{m}$. In SCA [Liu et al., 2016], $\mathbf{F}^{m}$ is discretized by a cluster-wise constant approximation $\left(\mathbf{F}^{m, k}\right)_{k=1 \ldots K}$. As a consequence, the microscopic first Piola-Kirchhoff stress tensor is also approximated cluster-wise $\left(\mathbf{P}^{m, k}\right)_{k=1 \ldots K}$, and Eq. (7) can be discretized as

$$
\mathbf{F}^{m, k}=-\sum_{k^{\prime}=1 \ldots K} \mathbb{D}^{0, k, k^{\prime}}:\left(\mathbf{P}^{m, k^{\prime}}-\mathbb{C}^{0}: \mathbf{F}^{m, k^{\prime}}\right)+\mathbf{F}^{0}, k=1 \ldots K
$$

where $\mathbb{D}^{0}$ is the interaction tensor defined by

$$
\begin{aligned}
\mathbb{D}^{0, k, k^{\prime}} & =\frac{1}{\left|\Omega_{0}^{m, k}\right|} \int_{\Omega_{0}^{m}} \chi^{k}(\boldsymbol{X}) \int_{\Omega_{0}^{m}} \chi^{k^{\prime}}\left(\boldsymbol{X}^{\prime}\right) \mathbb{G}^{0}\left(\boldsymbol{X}, \boldsymbol{X}^{\prime}\right) \mathrm{d} \boldsymbol{X}^{\prime} \mathrm{d} \boldsymbol{X} \\
& =\frac{1}{\left|\Omega_{0}^{m, k}\right|} \int_{\Omega_{0}^{m, k}}\left(\chi^{k^{\prime}} * \mathbb{G}^{0}\right)(\boldsymbol{X}) \mathrm{d} \boldsymbol{X} .
\end{aligned}
$$

The characteristic functions $\chi^{k}$ and $\chi^{k^{\prime}}$ are equal to 1 in, respectively, clusters $k$ and $k^{\prime}$, and 0 elsewhere. In the FFT-based numerical method [Moulinec \& Suquet, 1998], the periodic Green's operator $\mathbb{G}^{0}$ depends on $\mathbb{C}^{0}$, and is known in closed form in Fourier space. Because $\mathbb{C}^{0}$ is related to an isotropic linear elastic reference material, $\mathbb{G}^{0}$ can be expressed in Fourier space as a function of the reference Lamé parameters $\lambda^{0}$ and $\mu^{0}$. It is then obtained in real space by using the inverse FFT. In particular, Eq. (10) can be written in the form

$$
\begin{gathered}
\mathbb{D}^{0, k, k^{\prime}}=f^{1}\left(\lambda^{0}, \mu^{0}\right) \mathbb{D}^{1, k, k^{\prime}}+f^{2}\left(\lambda^{0}, \mu^{0}\right) \mathbb{D}^{2, k, k^{\prime}}, \\
\mathbb{D}^{i, k, k^{\prime}}=\frac{1}{\left|\Omega_{0}^{m, k}\right|} \int_{\Omega_{0}^{m, k}} \mathrm{FFT}^{-1}\left\{\operatorname{FFT}\left\{\chi^{k^{\prime}}\right\} \hat{\mathbb{G}}^{i}\right\}(\mathrm{X}) \mathrm{dX}, i=1,2 .
\end{gathered}
$$

The detailed expressions of $f^{1}, f^{2}, \hat{\mathbb{G}}^{1}$ and $\hat{\mathbb{G}}^{2}$ can be found in [Moulinec \& Suquet, 1998, Kabel et al., 2014, Liu et al., 2016] among others. Drastic computational cost reduction is enabled by SCA thanks to a reduced number of degrees of freedom by clustering, and by the fact that $\mathbb{D}^{1}$ and $\mathbb{D}^{2}$ can be precomputed in the training stage. Therefore, neither FFTs nor inverse FFTs are computed in the prediction stage, even if the reference material is changing.

In the present work, mixed boundary conditions are coupled to Eq. (9). Some components $\mathbf{F}_{i, j}^{m}$ of the average of the microscopic deformation gradient are set equal to their macroscopic counterparts from $\mathbf{F}_{i, j}^{M}$, 
and some other components $\mathbf{P}_{i, j}^{m}$ of the average of the microscopic first Piola-Kirchhoff stress tensor are set to zero. This can be done by adding the following conditions to Eq. (9):

$$
\left\{\begin{aligned}
\sum_{k=1 \ldots k}\left|\Omega_{0}^{m, k}\right| \mathbf{F}_{i, j}^{m, k} & =\left|\Omega_{0}^{m}\right| \mathbf{F}_{i, j}^{M}, & & (i, j) \in \mathcal{F} \\
\sum_{k=1 \ldots k}\left|\Omega_{0}^{m, k}\right| \mathbf{P}_{i, j}^{m, k} & =0, & & (i, j) \in\{1,2,3\}^{2} \backslash \mathcal{F}
\end{aligned}\right.
$$

where $\mathcal{F} \subset\{1,2,3\}^{2}$ is the set of components for which kinematic conditions are imposed.

As noted in [Liu et al., 2016], solutions of Eq. (9) depend on the choice of reference material. An optimal choice can be computed in the prediction stage by making the reference material consistent with the homogenized material. This means that the far field deformation gradient tensor $\mathbf{F}^{0}$ is an additional unknown that must be solved for in SCA [Liu et al., 2016], as opposed to the FFT-based numerical method where $\mathbf{F}^{0} \equiv \mathbf{F}^{M}$ [Kabel et al., 2014]. The self-consistent method consists in using a fixed-point iterative method where, at each step, the reference Lamé parameters $\lambda^{0}$ and $\mu^{0}$ are changed so that $\left\|\mathbf{P}^{M}-\mathbb{C}^{0}:\left(\mathbf{F}^{0}-\mathbf{I}\right)\right\|_{2}$ is minimized. This is presented in algorithm form in the Appendix.

\subsection{Summary}

To summarize, SCA is based on a voxel-wise discretization of the RVE domain, which is inherited from FFTbased numerical methods. The originality of SCA comes from the use of a K-means clustering algorithm in the training stage to cluster voxels based on a mechanistic a priori clustering criterion computed using a simple sampling of the loading space. This training stage also includes computing all voxel-wise and computationally expensive operations such as FFTs and inverse FFTs.

In the prediction stage, a self-consistent iterative algorithm is used to search for the optimal choice of reference Lamé parameters. At each iteration of this self-consistent loop, matrix assembly operations are accelerated because all voxel-wise operations have been precomputed in the training stage and already reduced to cluster-wise contributions. A Newton-Raphson iterative algorithm must be embedded within each self-consistent iteration as we are considering nonlinear materials, thus the discrete Lippmann-Schwinger equation is linearized.

The outputs from SCA are the microscopic variables' cluster-wise approximations, including the microscopic first Piola-Kirchhoff stress tensor. The latter can be used to predict void nucleation micromechanisms through stress-based fracture criteria as will be described in Sec. 4.

The main advantage of SCA over POD techniques is that in the prediction stage all operations are conducted cluster-wise in SCA instead of voxel-wise, including material integration and even fragmentation modeling.

\section{Numerical validation}

Before considering a specific application as proposed in Sec. 4, general ductile materials microstructure are modeled in this section both using a finite strain FFT-based numerical method and finite strain SCA. The goal is to validate the numerical convergence of SCA towards the reference result, and its capability to compute accurate predictions with a reduced complexity, as was shown for the small strain case in [Liu et al., 2016].

In the present large strain case, hyperelastic behavior is modeled both in the matrix and in the inclusions. A multiplicative von Mises plasticity model [Simo, 1992] with linear isotropic hardening is added to model the nonlinear response of the matrix. Inclusions are assumed to be brittle, which is a common assumption for hard phases in ductile materials. Their failure will be considered in Sec. 4. The model ductile material microstructure is shown in Fig. 3(a), and material properties are given in Tab. 1 for the matrix and the inclusions. The microstructure is discretized using $100 \times 100 \times 100$ voxels, which is sufficient to accurately predict the response with the FFT numerical method based on our preliminary calculations (not reported 
herein).

Table 1: Material parameters for the model ductile material

\begin{tabular}{lrrrc}
\hline Parameter & Matrix & Inclusion & Void & Units \\
\hline Young's Modulus & 70.0 & 400.0 & $70.0 \mathrm{e}-3$ & $\mathrm{GPa}$ \\
Poisson's Ratio & 0.33 & 0.2 & 0.0 & - \\
Yield Strength & 400 & - & - & $\mathrm{MPa}$ \\
Hardening Modulus & 1333 & - & - & $\mathrm{MPa}$ \\
\hline
\end{tabular}

The reference result is computed on the voxel mesh using the finite strain FFT based numerical method under unidirectional tension up to $25 \%$ applied logarithmic strain with strain increments of size 0.001 . The training database used for K-means clustering simply consists of the voxel-wise deformation gradient tensor extracted from the first increment of this reference simulation, which corresponds to a linear elastic analysis. In other words, the simulation used for training is identical in all aspects (mesh, geometry, material properties) to the one conducted in the prediction stage, except for loading conditions because only one strain increment is applied during training. This database can hence be constructed with a negligible computational cost. The K-means algorithm requires a predefined number of clusters, which is varied from $k_{1}=1,4,16,64,256$ in the matrix phase, and $k_{2}=1,1,4,13,26$ in the inclusion phase.

The comparison between the reference macroscopic stress/strain curve and those predicted by finite strain SCA is presented in Fig. 3 (b and c). For 16 clusters in the matrix and more, a very accurate prediction is obtained with finite strain SCA. This extends the validation conducted in [Liu et al., 2016] to large strains. To show the influence of clustering on computational complexity, a comparison of computation times is presented in Fig. 3(c). The computation time is shown to be reduced by four orders using SCA compared to the FFT based numerical method. This shows that SCA drastically reduces the complexity of microstructure calculations, based on a mechanistic clustering of voxels. 


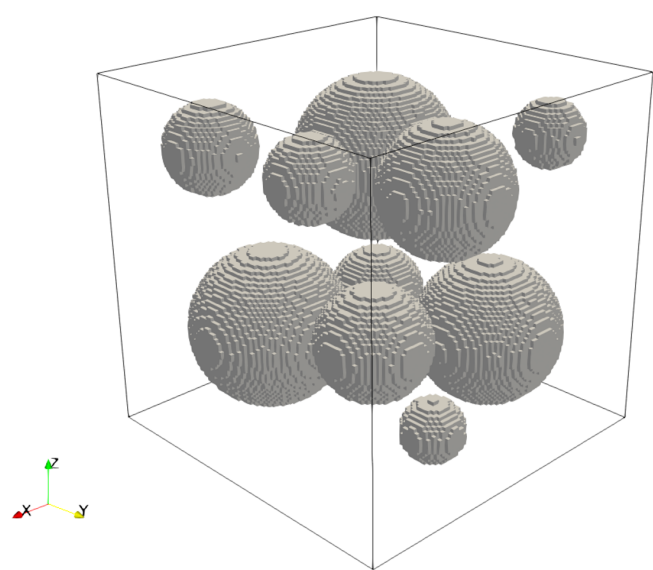

(a)

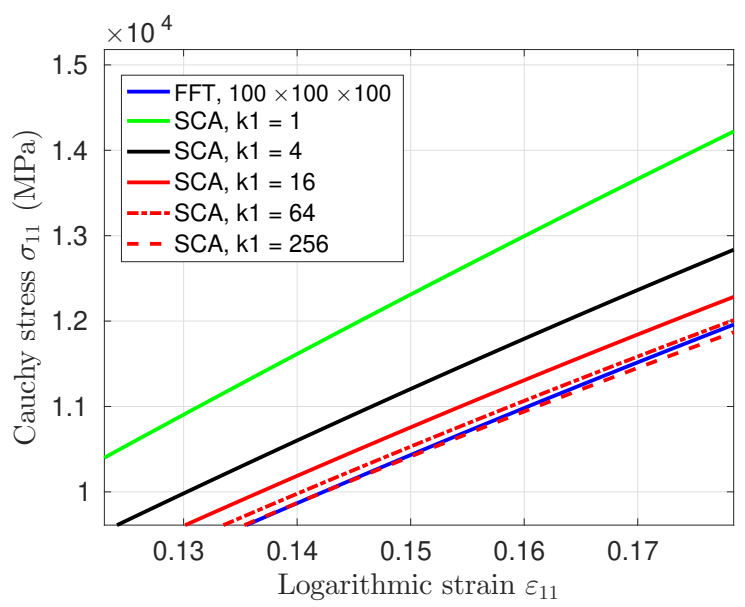

(c)

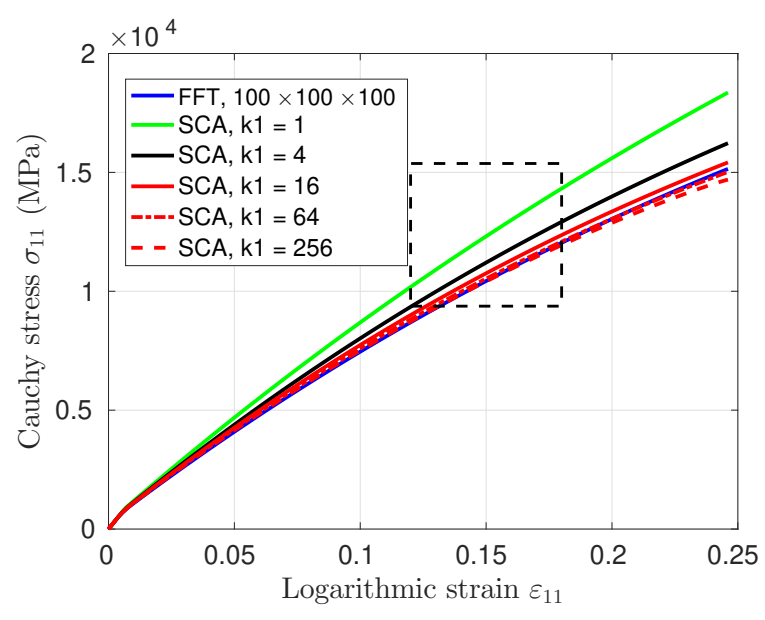

(b)

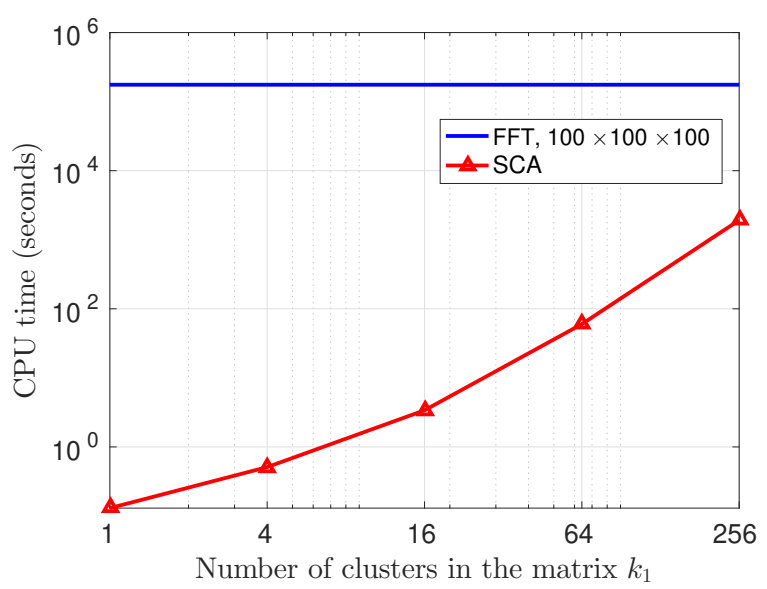

(d)

Figure 3: (a) Ductile composite with particle volume fraction of 20\%; (b) SCA converges fast to the FFT reference for the overall mechanical response of the RVE under unidirectional tension along the $x$ direction; (c) a closer look at the dashed rectangle area in (b) shows accurate prediction of SCA with only 16 clusters; (d) a CPU time saving of a factor of more than $10^{3}$ is achieved with SCA for the relatively well-converged number of clusters, compared with the FFT reference with a $100 \times 100 \times 100$ voxel mesh.

This interesting advantage of SCA is demonstrated in a second set of simulations where the inclusions in Fig. 3(a) are replaced by voids. Material properties for this porous ductile material are the same as those in Tab. 1, except that the stiffness of the voids is assumed to be $1 \%$ of that of the matrix. Loading is set to uniaxial tension to ensure that the stress state remains constant during the analysis.

The comparison between the reference macroscopic stress/strain curve and those predicted by finite strain SCA is presented in Fig. 4 (a and b). It can be seen that convergence is much slower for this example with voids, which features larger plastic strains than in the inclusions case. However, predictions are very close to the reference result.

The main advantage of SCA in terms of computation time is preserved, as shown in Fig. 4(b). As all SCA predictions are very close, one could use a very small number of clusters and obtain a good approximation of the reference result, resulting once again in a drastic reduction of computational complexity. 


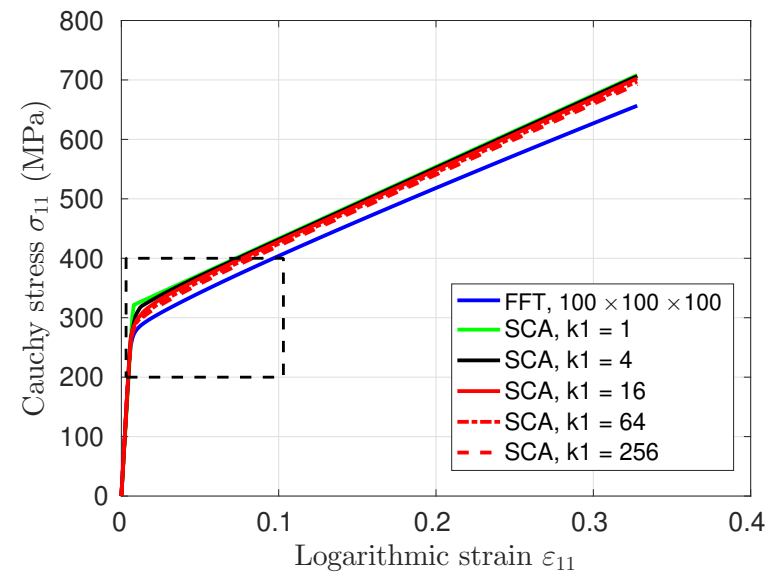

(a)

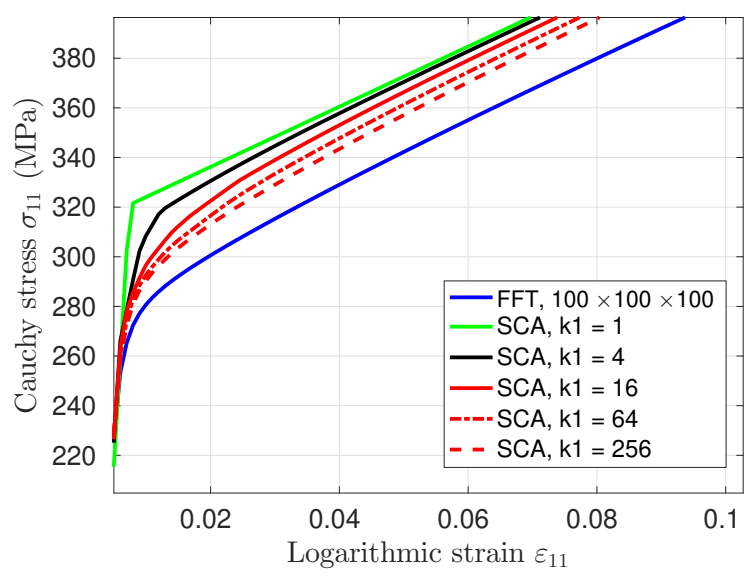

(b)

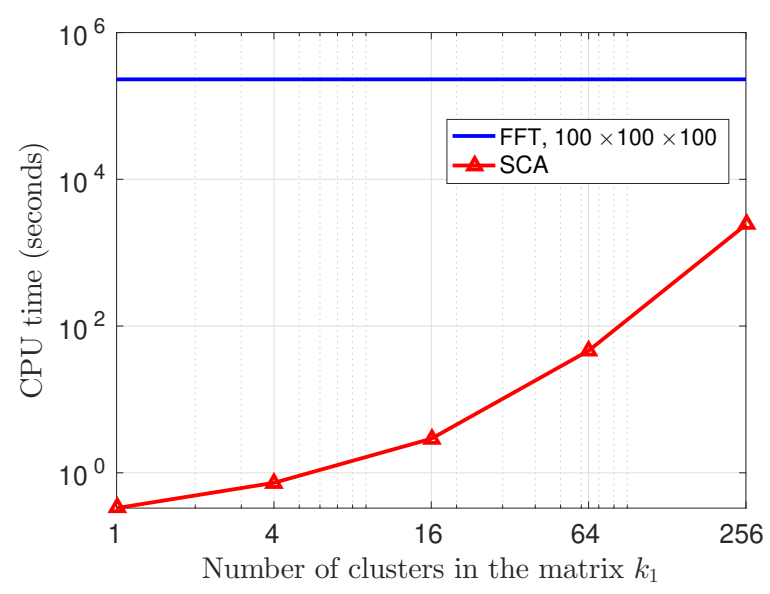

(c)

Figure 4: (a) SCA is fairly close to the FFT reference for the overall mechanical response of the porous RVE under uniaxial tension along the $x$ direction; (b) a closer look at dashed rectangle area in (a) shows the relatively slow convergence; (c) a CPU time saving of a factor of more than $10^{3}$ is achieved with SCA for relatively well-converged numbers of clusters, compared with the FFT reference with a $100 \times 100 \times 100$ voxel mesh.

These first simulations with the proposed finite strain SCA formulation are promising as predictions are close to the reference results for a drastically reduced computational complexity. However, this comparison is purely global: only averaged results are being assessed. At large plastic strain or for more complex loading conditions, very heterogeneous and localized strain fields may develop and would require improvements to the method. For instance, adaptive clustering techniques could be considered to update the clustering when plastic localization phenomena occur within the RVE.

\section{Application to Fatigue Strength Prediction of Cold Drawn NiTi Tubes}

In the continuation of Sec. 3, the objective of this section is to demonstrate the utility of a mechanical science of materials based on data science to predict process-structure-property-performance relationships. 
The chosen application is the prediction of the fatigue strength of cold drawn NiTi tubes as a function of drawing ratio and initial inclusion Aspect Ratio (AR).

NiTi tubes are formed using a series of hot and cold metal forming processes coupled to heat treatments [Frick et al., 2004, Toro et al., 2009, Lei et al., 2011, Sczerzenie et al., 2012]. They are used in the making of e.g. arterial stents and heart valve frames that undergo a large number of cyclic loads due to heart beats [Duerig et al., 1999, Elahinia et al., 2012, Moore et al., 2016, Adler et al., 2018]. A critical measure of a tube's performance is hence the fatigue strength of its material, which is itself a function of the material's fatigue life under different applied cyclic strain amplitudes. This fatigue life can be predicted using micromechanical simulations, which depend on the microstructural constitution of NiTi tubes. The latter is a consequence of forming processes, and in particular of the cold drawing step, which is the focus of the following study.

We propose simulating cold drawing at the microscale by applying biaxial compression to an initially debonded inclusion embedded within a NiTi matrix. In order to predict the evolution of this microstructure during cold drawing, the finite strain SCA theory introduced in Sec. 2 is completed with a fragmentation model in Subsec. 4.1. The microstructure is extracted at different stages of this drawing model and used as input to a data-driven fatigue life prediction model developed in previous studies [Liu et al., 2018b, Kafka et al., 2018] and described briefly in Subsec. 4.2. The transfer of the microstructure morphology from the drawing model to the fatigue life prediction model requires a displacement reconstruction and microstructure interpolation step that is described in Subsec. 4.3. Process-structure-propertyperformance predictions obtained using this data science approach are presented in 4.4.

\subsection{Cold drawing model}

Using the self-consistent scheme presented in Subsec. 2.3, the discrete Lippmann-Schwinger equation (9) can be solved with mixed boundary conditions (12) and appropriate constitutive models for the microstructure's constituents. Constitutive models and material parameters are kept identical to those used in Sec. 3 and reported in Tab. 1, but they are completed by an inclusion fragmentation model. In addition, inclusions are assumed to be initially debonded as a result of high shear stresses developing early at the inclusion/matrix interface during cold drawing, similarly to the cold extrusion process [McVeigh \& Liu, 2006].

Inclusions fragmentation is modeled using a Tresca yield criterion averaged inclusion-wise, following a regularization technique used in a previous work [Shakoor et al., 2018] and coupled to a size-effect criterion. The Tresca criterion defines the shear stress $\sigma_{\text {Tresca }}$ as

$$
\sigma_{\text {Tresca }}=\frac{1}{2} \max \left|\sigma_{1}-\sigma_{2}\right|,\left|\sigma_{2}-\sigma_{3}\right|,\left|\sigma_{3}-\sigma_{1}\right|
$$

where $\sigma_{1}, \sigma_{2}, \sigma_{3}$ are the cluster-wise constant principal stresses computed within each cluster of the inclusion phase. The shear stress $\sigma_{\text {Tresca }}$ is then averaged inclusion-wise, or inclusion fragment-wise if the inclusion has already broken-up, and that averaged value $\bar{\sigma}_{\text {Tresca }}$ is compared to the inclusion shear strength $\sigma_{\text {Tresca }}^{c}$. The equivalent radius of this inclusion or inclusion fragment $r$ is also compared to a critical size parameter $r^{c}$. If the shear strength has been reached and $r \geq r_{c}$, the inclusion cluster containing the $\sigma_{\text {Tresca-weighted }}$ barycenter of that inclusion or inclusion fragment is turned into void, as illustrated in Fig. 5. In practice, this consists in reducing its Young modulus to a 1000th of its initial value over several load increments. This procedure is carried out at the end of each load increment of the finite strain SCA simulation, once Eqs. (9) and (12) have been solved using the self-consistent scheme. As revealed in Fig. 5, the orientation of the fragmentation crack is predefined and is initially orthogonal to the drawing direction. The fracture criterion parameters are given in Tab. 2. 
Table 2: Fracture criterion parameters for the cold drawing model

\begin{tabular}{lrc}
\hline Parameter & Value & Units \\
\hline Shear Strength & 3000 & $\mathrm{MPa}$ \\
Critical inclusion size & 0.17 & $1 / \mathrm{RVE}$ size \\
\hline
\end{tabular}

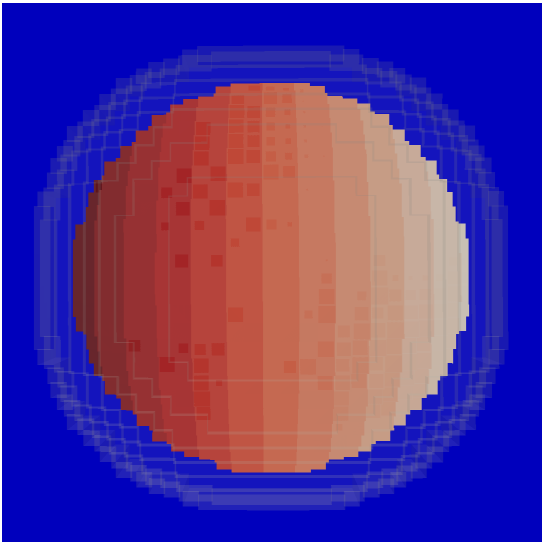

(a)

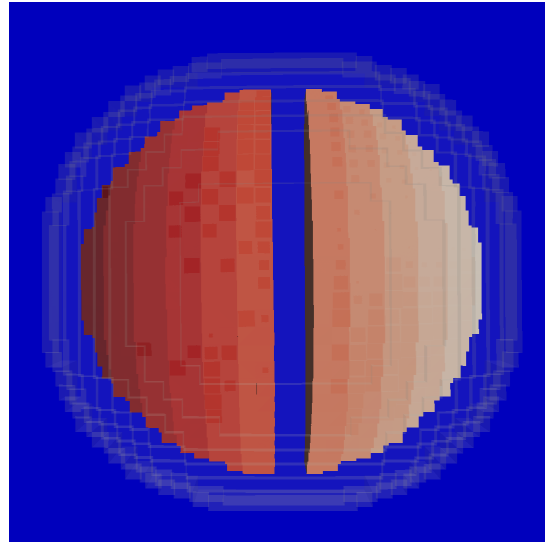

(b)

Figure 5: Clusters (shades of red) within the debonded inclusion: (a) before fragmentation, (b) after fragmentation with the missing cluster turned into void. Drawing direction is horizontal.

\subsection{Fatigue Life Prediction model}

High-cycle fatigue life can be estimated based on local plastic deformation predicted under cyclic loading, where these deformations are computed using the SCA outlined above. Since cyclic strain amplitudes are low, typically below $1 \%$ reversed strain, a small strain formulation can be used. An appropriate micro-scale material law - crystal plasticity $(\mathrm{CP})$ - is solved cluster-wise to obtain the cyclic change in plastic shear strain $\left(\Delta \gamma_{p}\right)$ and stress normal $\left(\sigma_{n}\right)$ to that strain in the matrix material. The peak value of these variables reach cyclic steady-state rapidly, typically within 3 or 4 loading cycles. From these, a scalar value called the Fatigue Indicating Parameter (FIP) can be defined, which quantifies the fatigue driving force at any location. Here, we adopt the Fatemi-Socie FIP, defined by

$$
\mathrm{FIP}=\frac{\Delta \gamma_{p}}{2}\left(1+\kappa \frac{\sigma_{n}}{\sigma_{y}}\right)
$$

Originally developed by Fatemi and Socie [Fatemi \& Socie, 1988], this FIP is a critical plane approach based upon the plane of maximum normal stress, $\sigma_{n}$, normalized by the material yield stress $\left(\sigma_{y}\right)$ and a material dependent parameter $\kappa$, which controls the influence of normal stress (here $\kappa$ will be taken as 0.55). When used with SCA and a CP law, the FIP in each cluster is computed cluster-wise from plastic strain and stress across time increments using a simple search to maximize the plastic strain, and thus identify the critical plane. The maximum, saturated FIP $\left(\mathrm{NFIP}_{\max }\right)$ can be correlated to the number of fatigue incubation cycles $\left(N_{i n c}\right)$ a microstructure can withstand using a Coffin-Manson parameterization. By computing $N_{i n c}$ for a number of different strain amplitudes to generate a strain-life curve, the fatigue strength (strain amplitude at which a given number of cycles can be reached) can be computed through interpolation or fitting.

The CP material law from [Moore et al., 2016], calibrated to capture the hardening response of the B2 phase of NiTi, is used. Post-yield hardening is computed with a backstress term that accounts for direct and dynamic hardening. Consistent with the worst-case or nearly worst-case approximation for the material phase, and the lack of crystallographic information from the cold drawing model, the matrix is assumed to 
be composed of a single grain oriented such that its Schmid factor is maximized. The hard inclusion phase (representing oxides or carbides) is taken to be linear-elastic with an elastic modulus 10 times that of the matrix material. This process follows the CPSCA formulation outlined in [Liu et al., 2018b], which integrates the CP material law within SCA, and the fatigue prediction method shown for synthetic microstructures in [Kafka et al., 2018]. Indeed, the same model parameters are used for CP, FIP and Coffin-Manson as are shown in [Kafka et al., 2018].

\subsection{Transfer of microstructure from cold drawing model to fatigue life predic- tion model}

The fatigue life prediction model relies on CPSCA and hence on an underlying voxel mesh. In order to use this model, the deformed and fragmented microstructures from the drawing simulation results need to be transferred to a new voxel mesh.

The first step is to reconstruct the microscopic displacement vector field, since both FFT-based numerical methods and SCA only solve for the microscopic deformation gradient tensor. This is done using a simple Taylor expansion, or forward finite difference formula, which computes the displacements at all nodes of the voxel mesh from the microscopic deformation gradients inside voxels. This computation starts at some arbitrary corner of the RVE domain where the displacement is fixed to zero, which is in agreement with FE-based linear homogenization implementations [Geers et al., 2010].

Once the displacement vector field has been reconstructed, the mesh can be deformed by adding these displacements to node coordinates, as shown in Fig. 6(a,b). Finally, the phase tags (matrix, inclusion, void) are transferred from this deformed mesh to a new voxel mesh of the deformed RVE domain using a simple voxel-wise constant interpolation. As a result, the new voxel mesh is compatible with FFT-based numerical methods and SCA, but embeds phase tags that correspond to a cold drawn microstructure. Fatigue life predictions can be computed on this new voxel mesh using the CPSCA method, as shown in Fig. 6(c). 


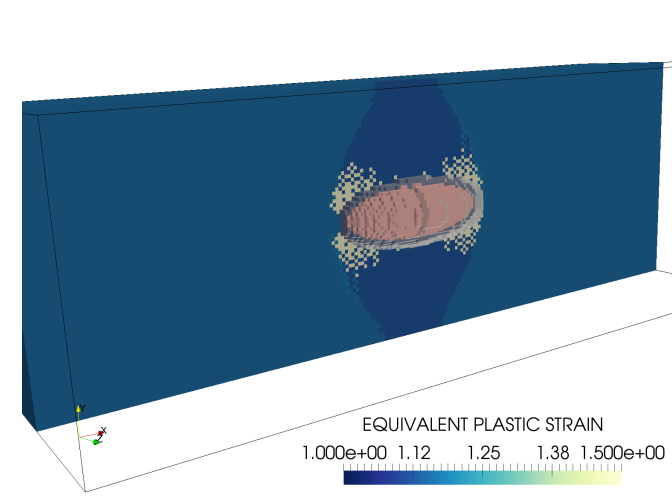

(a)

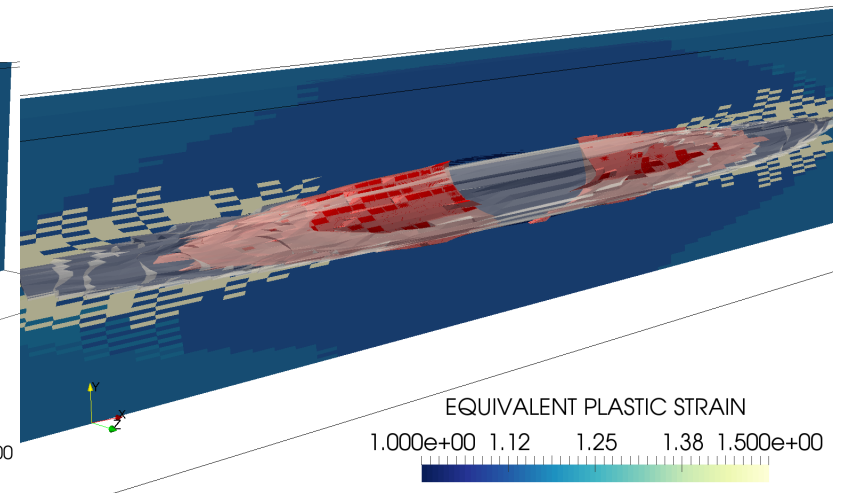

(b)

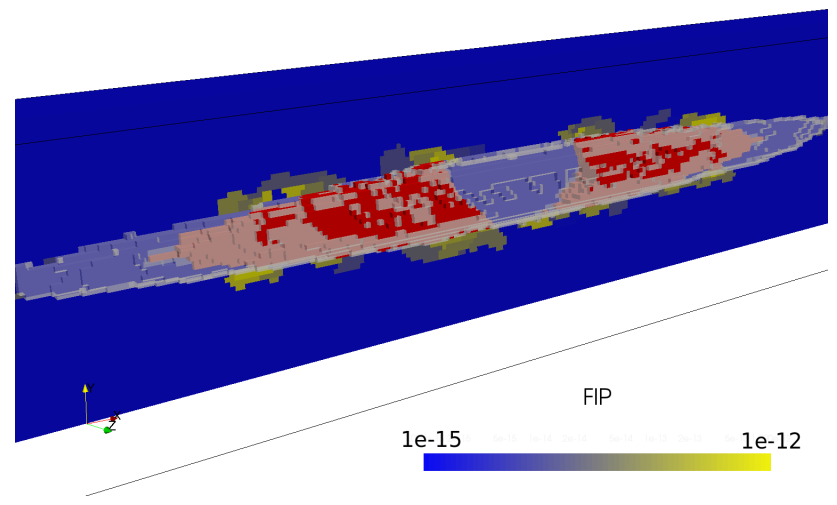

(c)

Figure 6: Cold drawing and FIP computation results showing the particle fragments in red, the voids in light gray, and: (a) the equivalent plastic strain in the matrix in undeformed configuration, (b) the equivalent plastic strain in the matrix in deformed configuration after displacement reconstruction, (c) the FIP in the matrix on a new voxel mesh of the deformed configuration after mesh transfer. Note that the three figures are not in the same spatial scale, as section height in (b,c) is reduced by $45 \%$ compared to (a).

\subsection{Fatigue strength predictions}

Three different initial conditions for the drawing model were considered, representing possible variability in the quality and degree of processing in the feed stock used for cold drawing. Each condition includes a single, ellipsoidal, debonded inclusion centered in the matrix with a different AR in the load direction. The cross-sectional area (i.e. the minor axis of the ellipsoid) of the initial configuration is kept constant, and the length (in the drawing direction) changed. By doing this, we study the influence of AR (mean curvature) on drawing, fragmentation and subsequent fatigue life. These three different cases are deformed to up to $60 \%$ section height reduction by applying biaxial compression with a stress-free third axis. For each case, at every $5 \%$ height reduction the procedure outline in Subsec. 4.3 is performed and the fatigue strength is computed. In order to compute the fatigue strength, fatigue lives at strain amplitudes ranging between $0.36 \%$ and $0.54 \%$ in increments of $0.06 \%$ were computed, and the strain amplitude required to reach $10^{7}$ cycles was estimated using a power-law fit to that data. Triangle waveforms with loading rate $0.1 / s$ were used throughout to apply fully reversed tension-compression (strain ratio, $R=-1$ ) fatigue loading. 
The results from this parametric study are shown in Fig. 7. In the center of the figure, fatigue strengththe maximum strain amplitude at which a specified number of cycles can be obtained-is plotted versus the section reduction resulting from drawing. At each point in section reduction and for each AR the fatigue strength at $10^{7}$ cycles is computed using the procedure outlined above. Cross-sections of the volume are given at six different points in order to visualize and analyze the process by which the fatigue strength changes with reduction percent. The drawing model captures the overall trend of an increase in fatigue life with increasing height reduction, particularly for the highest AR. A large increase in the fatigue life is noted when the AR 3 particle fragments, between $40 \%$ and $45 \%$ reduction. The pre-fragmentation configuration is shown in the upper right-hand subset to Fig. 7, and the post-fragmentation configuration was shown previously in Fig. 6(c). The distribution of FIP changes noticeably between these two states, with the field much more concentrated at the interfaces before fragmentation and more distributed throughout the volume after fragmentation. Up to $60 \%$ height reduction neither AR 1 nor 2 fragment, and no substantial decrease in life is noted. This is consistent with experimental experience, where relatively large reductions are often required to achieve large gains in fatigue performance. Higher reduction percents or different material properties would be required to fragment these cases.

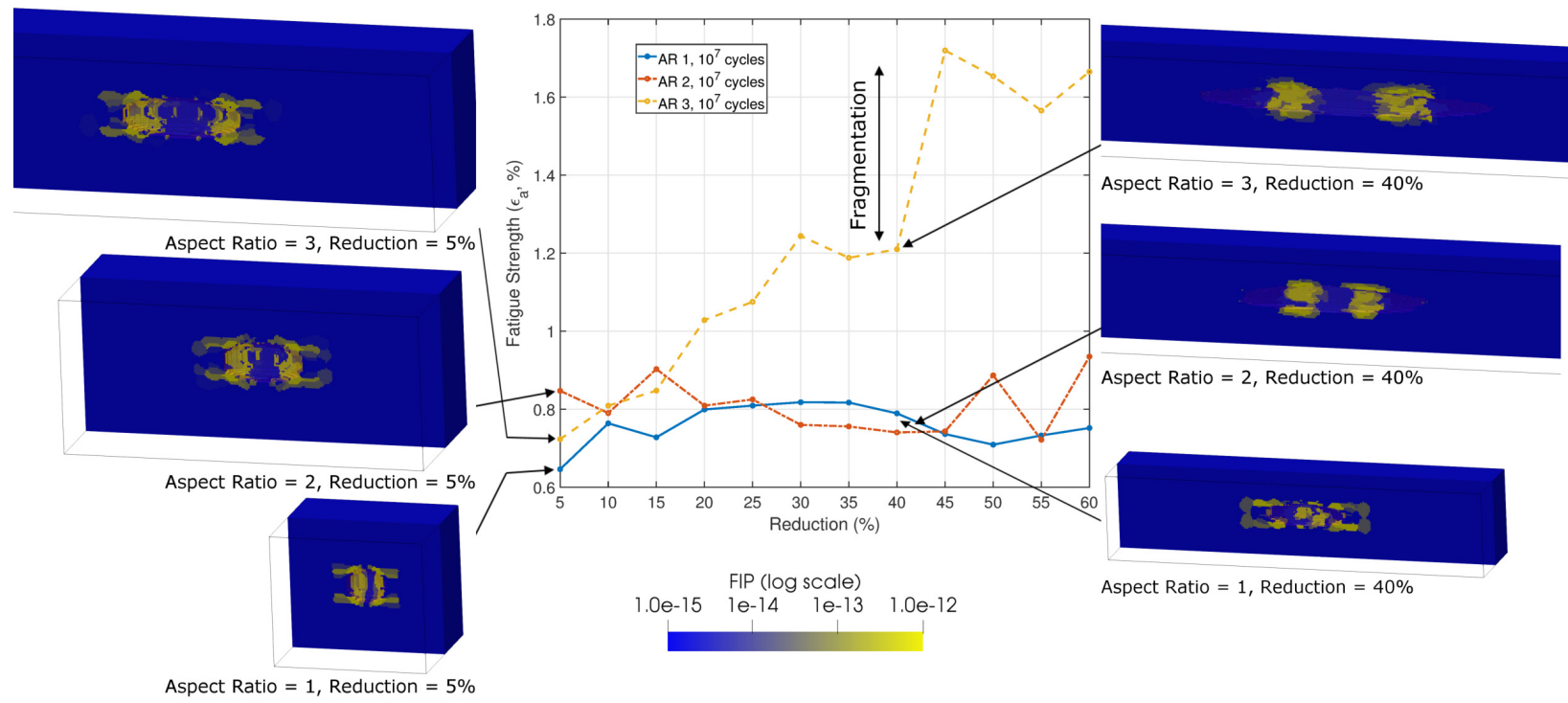

Figure 7: Fatigue strength at $10^{7}$ cycles as a function of cold drawing section reduction for 1:1:1, 2:1:1, and 3:1:1 initial AR (x:y:z). Fragmentation occurs between $40 \%$ and $45 \%$ reduction for AR 3, resulting in a jump in fatigue strength

\section{Conclusions}

A general formulation of data science approaches for mechanical science of materials has been presented in this paper. This general formulation consists in reducing the complexity of process-structure-propertyperformance prediction methods using unsupervised learning methods on a training database of high-fidelity simulations. This is evidenced in the case of a training database composed of RVE simulations results computed under various loading conditions using the FE method or FFT-based numerical methods. Dimension reduction leads to a compressed RVE model where nodes and voxels are replaced by either modes or clusters depending on the supervised learning method used for data compression.

In the prediction stage, supervised learning methods or mechanistic equations are solved using the compressed RVE. For instance, POD can be used to solve the Cauchy equation using a compressed FE discretization where the complexity depends on the number of modes instead of the number of nodes. Similarly, K-means 
clustering can be used to solve the Lippmann-Schwinger equation with a complexity depending on the number of clusters instead of the number of voxels. The interesting advantage of this later approach over the former is that it reduces both the complexity of mechanistic equations solution and material integration.

The solution of the Lippmann-Schwinger equation using a clustered discretization requires a self-consistent scheme that has been extended to finite strain elastoplastic materials and coupled to micromechanical void nucleation models in this paper. As a result, microstructure evolutions due to large plastic strains have been captured during the cold drawing of NiTi tubes. These microstructure evolutions have been related to the fatigue life and then the fatigue strength of NiTi tubes using a second data science based approach developed in a previous work. Therefore, it has been demonstrated that the proposed data science formulation for mechanical science of materials can predict process-structure-property-performance with a reduced complexity.

\section{Conflicts of Interest}

The authors declare that there is no conflict of interest regarding the publication of this paper.

\section{Funding Statement}

WKL, CY and MS were supported by the Center for Hierarchical Materials Design (CHiMaD) under Grant Nos. 70NANB13H194 and 70NANB14H012; WKL is also thankful to the AFOSR for support in the preliminary stages of a newly funded proposal and the National Science Foundation (NSF) Cyber-Physical Systems (CPS) for funding under grant No. CPS/CMMI-1646592 and Mechanics of Materials and Structures (MOMS) under the newly funded grant No. MOMS/CMMI-1762035. OLK acknowledges the support of the NSF Graduate Research Fellowship under Grant No. DGE-1324585a.

\section{Appendix}

The finite strain SCA algorithm is given in Alg. 1. The fracture criteria introduced in Sec. 4 are considered only once a converged solution has been computed using this algorithm. The convergence criterion is a tolerance on the variation on $\mathbf{F}^{m}$ between two self-consistent scheme iterations. 


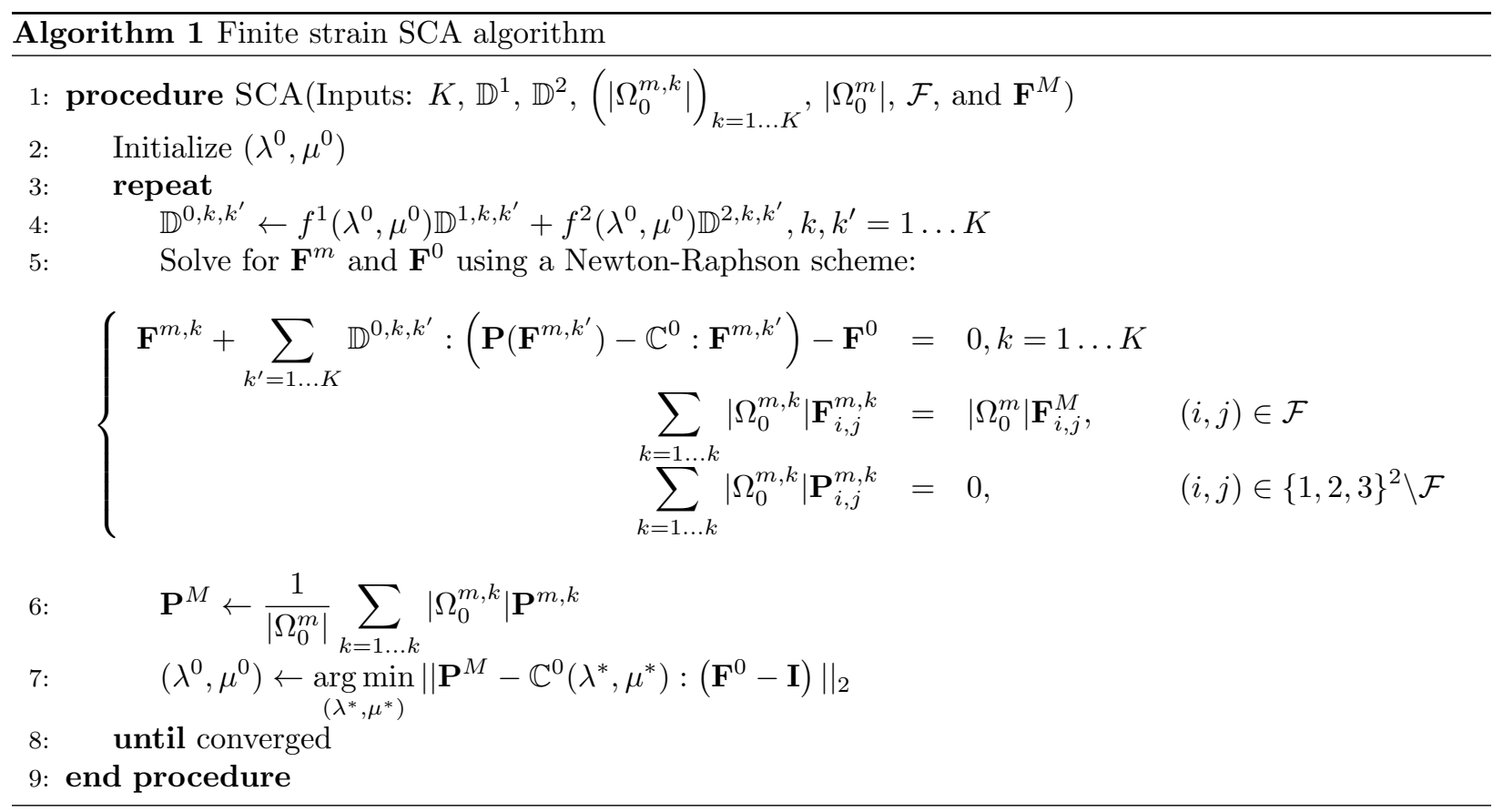

\section{References}

[Adler et al., 2018] Adler, P., Frei, R., Kimiecik, M., Briant, P., James, B., \& Liu, C. (2018). Effects of Tube Processing on the Fatigue Life of Nitinol. Shape Memory and Superelasticity, 4(1), 197-217.

[Bessa et al., 2017] Bessa, M. A., Bostanabad, R., Liu, Z., Hu, A., Apley, D. W., Brinson, C., Chen, W., \& Liu, W. K. (2017). A framework for data-driven analysis of materials under uncertainty: Countering the curse of dimensionality. Computer Methods in Applied Mechanics and Engineering, 320, 633-667.

[Chinesta et al., 2013] Chinesta, F., Leygue, A., Bordeu, F., Aguado, J. V., Cueto, E., Gonzalez, D., Alfaro, I., Ammar, A., \& Huerta, A. (2013). PGD-Based Computational Vademecum for Efficient Design, Optimization and Control. Archives of Computational Methods in Engineering, 20(1), 31-59.

[Duerig et al., 1999] Duerig, T., Pelton, A., \& Stöckel, D. (1999). An overview of nitinol medical applications. Materials Science and Engineering: A, 273-275, 149-160.

[Dvorak, 1992] Dvorak, G. J. (1992). Transformation Field Analysis of Inelastic Composite Materials. Proceedings of the Royal Society A: Mathematical, Physical and Engineering Sciences, 437(1900), 311-327.

[Elahinia et al., 2012] Elahinia, M. H., Hashemi, M., Tabesh, M., \& Bhaduri, S. B. (2012). Manufacturing and processing of NiTi implants: A review. Progress in Materials Science, 57(5), 911-946.

[Fatemi \& Socie, 1988] Fatemi, A. \& Socie, D. F. (1988). A Critical Plane Approach to Multiaxial Fatigue Damage Including out-of-Phase Loading. Fatigue Fract. Eng. Mater. Struct.,, 11, 149.

[Frick et al., 2004] Frick, C. P., Ortega, A. M., Tyber, J., Gall, K., \& Maier, H. J. (2004). Multiscale structure and properties of cast and deformation processed polycrystalline NiTi shape-memory alloys. Metallurgical and Materials Transactions A, 35(7), 2013-2025.

[Geers et al., 2010] Geers, M. G. D., Kouznetsova, V. G., \& Brekelmans, W. A. M. (2010). Computational homogenization. In Multiscale Modelling of Plasticity and Fracture by Means of Dislocation Mechanics (pp. 327-394). 
[Goury et al., 2016] Goury, O., Amsallem, D., Bordas, S. P. A., Liu, W. K., \& Kerfriden, P. (2016). Automatised selection of load paths to construct reduced-order models in computational damage micromechanics: from dissipation-driven random selection to Bayesian optimization. Computational Mechanics, 58(2), $213-234$.

[Hambli et al., 2011] Hambli, R., Katerchi, H., \& Benhamou, C.-L. (2011). Multiscale methodology for bone remodelling simulation using coupled finite element and neural network computation. Biomechanics and Modeling in Mechanobiology, 10(1), 133-145.

[Kabel et al., 2014] Kabel, M., Böhlke, T., \& Schneider, M. (2014). Efficient fixed point and Newton-Krylov solvers for FFT-based homogenization of elasticity at large deformations. Computational Mechanics, 54(6), $1497-1514$.

[Kafka et al., 2018] Kafka, O. L., Yu, C., Shakoor, M., Liu, Z., Wagner, G. J., \& Liu, W. K. (2018). DataDriven Mechanistic Modeling of Influence of Microstructure on High-Cycle Fatigue Life of Nickel Titanium. JOM, (2).

[Kerfriden et al., 2011] Kerfriden, P., Gosselet, P., Adhikari, S., \& Bordas, S. P. A. (2011). Bridging proper orthogonal decomposition methods and augmented Newton-Krylov algorithms: An adaptive model order reduction for highly nonlinear mechanical problems. Computer Methods in Applied Mechanics and Engineering, 200(5-8), 850-866.

[Ladevèze et al., 2010] Ladevèze, P., Passieux, J.-C., \& Néron, D. (2010). The LATIN multiscale computational method and the Proper Generalized Decomposition. Computer Methods in Applied Mechanics and Engineering, 199(21-22), 1287-1296.

[Le et al., 2015] Le, B. A., Yvonnet, J., \& He, Q.-C. (2015). Computational homogenization of nonlinear elastic materials using neural networks. International Journal for Numerical Methods in Engineering, 104(12), 1061-1084.

[Lei et al., 2011] Lei, X., Rui, W., \& Yong, L. (2011). The optimization of annealing and cold-drawing in the manufacture of the Ni-Ti shape memory alloy ultra-thin wire. The International Journal of Advanced Manufacturing Technology, 55(9-12), 905-910.

[Liang et al., 2002] Liang, Y. C., Lee, H. P., Lim, S. P., Lin, W. Z., Lee, K. H., \& Wu, C. G. (2002). Proper Orthogonal Decomposition and its applications - Part I: Theory. Journal of Sound and Vibration, 252(3), $527-544$.

[Lieu et al., 2006] Lieu, T., Farhat, C., \& Lesoinne, M. (2006). Reduced-order fluid/structure modeling of a complete aircraft configuration. Computer Methods in Applied Mechanics and Engineering, 195(41-43), $5730-5742$.

[Liu et al., 2016] Liu, Z., Bessa, M. A., \& Liu, W. K. (2016). Self-consistent clustering analysis: An efficient multi-scale scheme for inelastic heterogeneous materials. Computer Methods in Applied Mechanics and Engineering, 306, 319-341.

[Liu et al., 2018a] Liu, Z., Fleming, M., \& Liu, W. K. (2018a). Microstructural material database for selfconsistent clustering analysis of elastoplastic strain softening materials. Computer Methods in Applied Mechanics and Engineering, 330, 547-577.

[Liu et al., 2018b] Liu, Z., Kafka, O. L., Yu, C., \& Liu, W. K. (2018b). Data-Driven Self-consistent Clustering Analysis of Heterogeneous Materials with Crystal Plasticity. volume 46 of Computational Methods in Applied Sciences (pp. 221-242). Springer International Publishing.

[McVeigh \& Liu, 2006] McVeigh, C. \& Liu, W. K. (2006). Prediction of central bursting during axisymmetric cold extrusion of a metal alloy containing particles. International Journal of Solids and Structures, 43(10), 3087-3105. 
[Moore et al., 2016] Moore, J. A., Frankel, D., Prasannavenkatesan, R., Domel, A. G., Olson, G. B., \& Liu, W. K. (2016). A crystal plasticity-based study of the relationship between microstructure and ultra-highcycle fatigue life in nickel titanium alloys. Int. J Fatigue,, 91, 183.

[Moulinec \& Suquet, 1998] Moulinec, H. \& Suquet, P. (1998). A numerical method for computing the overall response of nonlinear composites with complex microstructure. Computer Methods in Applied Mechanics and Engineering, 157(1-2), 69-94.

[Ryckelynck, 2005] Ryckelynck, D. (2005). A priori hyperreduction method: an adaptive approach. Journal of Computational Physics, 202(1), 346-366.

[Ryckelynck \& Missoum Benziane, 2010] Ryckelynck, D. \& Missoum Benziane, D. (2010). Multi-level A Priori Hyper-Reduction of mechanical models involving internal variables. Computer Methods in Applied Mechanics and Engineering, 199(17-20), 1134-1142.

[Sczerzenie et al., 2012] Sczerzenie, F., Vergani, G., \& Belden, C. (2012). The Measurement of Total Inclusion Content in Nickel-Titanium Alloys. Journal of Materials Engineering and Performance, 21(12), $2578-2586$.

[Shakoor et al., 2018] Shakoor, M., Bernacki, M., \& Bouchard, P.-O. (2018). Ductile fracture of a metal matrix composite studied using 3D numerical modeling of void nucleation and coalescence. Engineering Fracture Mechanics, 189, 110-132.

[Simo, 1992] Simo, J. (1992). Algorithms for static and dynamic multiplicative plasticity that preserve the classical return mapping schemes of the infinitesimal theory. Computer Methods in Applied Mechanics and Engineering, 99(1), 61-112.

[Tang et al., 2018] Tang, S., Zhang, L., \& Liu, W. K. (2018). From virtual clustering analysis to selfconsistent clustering analysis: a mathematical study. Computational Mechanics, (pp. 1-18).

[Toro et al., 2009] Toro, A., Zhou, F., Wu, M. H., Van Geertruyden, W., \& Misiolek, W. Z. (2009). Characterization of Non-Metallic Inclusions in Superelastic NiTi Tubes. Journal of Materials Engineering and Performance, 18(5-6), 448-458.

[Yvonnet \& He, 2007] Yvonnet, J. \& He, Q. C. (2007). The reduced model multiscale method (R3M) for the non-linear homogenization of hyperelastic media at finite strains. Journal of Computational Physics, $223(1), 341-368$.

[Zhang \& Friedrich, 2003] Zhang, Z. \& Friedrich, K. (2003). Artificial neural networks applied to polymer composites: a review. Composites Science and Technology, 63(14), 2029-2044. 